\section{Integrating Interventions and Services}

\section{Research: Progress and Prospects}

Michael A. Southam-Gerow, Virginia Commonwealth

University

Heather L. Ringeisen and Joel T. Sherrill, National Institute of Mental Health

Although interventions and services research paradigms have distinct historical roots and methodologic traditions, both aim to improve mental health services for adults and youth. This article introduces a series of articles that represent examples of innovative and integrative (i.e., integration of services and interventions research) research efforts. This work involves an integration of the interventions and services research paradigms to address the difficult questions related to improving mental health services in diverse settings. Each of the four articles takes a distinctly different path in bringing together the interventions and services research traditions, with foci ranging from (a) using psychotherapy measurement tools to assess the content of usual care practice in collaboration with providers, (b) adapting randomized clinical trial design to fit nonmental healthcare settings while addressing relevant outcomes, (c) understanding and incorporating patient preferences into treatment research, and (d) identifying organization-level variables relevant to interventions

Address correspondence to Michael A. Southam-Gerow, Virginia Commonwealth University, Department of Psychology, 808 W. Franklin St. Box 842018, Richmond, Virginia 232842018. E-mail: masouthamger@vcu.edu. development and implementation. In the introductory article, we provide a brief précis concerning the interventions and services research traditions, highlight how each of the four articles illustrates an innovative integration of interventions and services research, and discuss additional future directions beyond the work introduced in this series.

Key words: mental health research in primary care, organizational factors, patient preferences, services research, treatment research, usual care. [Clin Psychol Sci Prac 13: 1-8, 2006]

Mental health interventions and services research paradigms were developed with unique goals: one, to optimize therapeutics, and the other, to understand and optimize care delivery. Within the past 20 years, there has been tremendous growth across each area of mental health science. For instance, interventions research has led to the accumulation of a growing evidence base in support of the efficacy of treatments for mental disorders (Chambless \& Ollendick, 2001; Hoagwood, Burns, Kiser, Ringeisen, \& Schoenwald, 2001; Kazdin \& Weisz, 2003; Kendall, 1998; Nathan \& Gorman 2002). Meanwhile, services research has documented remarkable rates of unmet mental health service needs (Kataoka, Zhang, \& Wells, 2002; Kessler et al., 2001; Leaf et al., 1996; The WHO World Mental Health Survey Consortium, 2004) and the apparent reliance of mental health consumers on nontraditional care systems (e.g., Burns et al., 1995; Wang, Berglund, \& Kessler, 2003). Despite this growing knowledge, it appears that efficacious interventions are not being systematically used within communities (e.g., Norquist, Lebowitz, \& Hyman, 1999; Street, Niederehe, \& Lebowitz, 
2000; Weiss, Catron, \& Harris, 2000; Weisz, Weiss, \& Donenberg, 1992), and documentations of unmet need appear to have had limited impact on increased access to mental health services (Ringel \& Sturm, 2001). Thus, although a great deal has been learned through interventions and services research paradigms, this knowledge has not optimally translated into improved everyday practice therapeutics or efficient, high-quality care delivery (e.g., National Institutes of Health \& National Institute of Mental Health, 1999).

The goal of this special issue is to review the rationale for and opportunities presented by efforts to integrate interventions and services research foci (and methods). First, we briefly provide a historical overview of the contributions of interventions and services research traditions. Next, we discuss expanding the utility and generalizability of clinical research. Subsequently, we introduce the articles within the special issue as examples of successful efforts at the cross-fertilization between the interventions and services research areas. Finally, we comment on the potential of further transaction between interventions and services researchers and discuss some future directions and remaining opportunities. We contend that more collaboration between interventions and services scientists will increase the rigor and relevance of both research agendas, as well as expedite the improvement of care for individuals experiencing mental health problems.

\section{HISTORICAL FOCUS OF INTERVENTIONS AND SERVICES RESEARCH}

Mental health services research and clinical interventions research ultimately aim to improve health care for individuals with mental illness. Each research focus, however, evolved from relatively distinct origins with different methodologic traditions and different primary foci (Donenberg, Lyons, \& Howard, 1999). Clinical interventions research strives to develop, refine, and optimize interventions that ameliorate mental health problems. Led by the fields of psychology and psychiatry, interventions research has most often employed a clinical trials research design (e.g., Friedman, Furberg, \& DeMets, 1998) to test the safety, feasibility, and efficacy of a treatment for a specific population (e.g., adults with major depressive disorder). Typically, state-of-the-art interventions research designs involve randomization of patients to treatment conditions (i.e., randomized clinical trials [RCTs]) to allow for comparison of the experimental intervention to alternative therapies or control conditions as appropriate (see Westen, Novotny, \& Thompson-Brenner, 2004 for a different perspective). Variations such as dismantling or additive designs are additionally used to examine active ingredients of interventions or mechanisms of action; parametric studies (e.g., examinations of doses) are often used to investigate strategies for optimizing therapeutics.

Mental health services research has its methodologic roots in psychiatric epidemiology and the behavioral sciences. Early mental health services research described the processes of care for individuals with mental illness and the interaction of patient characteristics with those of diverse healthcare settings (Mechanic, 1989). In a historical overview of mental health services research, Mechanic (1989) described some hallmark studies within the field. Early examples of such work include an ethnography of the chronically mentally ill residing in a community model program (Estroff, 1981), as well as epidemiologic studies of patient referral patterns and psychiatric care placement (e.g., Hollingshead \& Redlich, 1958). More recent mental health services research has examined the influence of financing (e.g., structure of insurance programs) or organizational strategies (e.g., allocation of staff responsibilities) on care quality or patient outcomes or both.

Interventions and services research occurs within the multilayer and dynamic context of mental health service delivery (see Figure 1, adapted from Schoenwald \& Hoagwood, 2001). This context has been described as

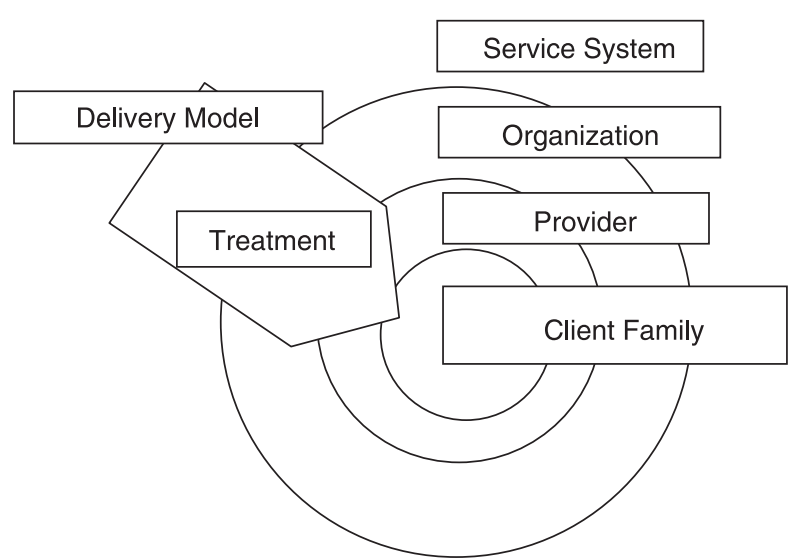

Figure 1. Layers of the mental health service delivery context. (Adapted from Schoenwald \& Hoagwood, 2001, Figure 1, page 4. Copyright 2001 by Civic Research Institute. Adapted with permission.) 
consisting of client-level factors (e.g., symptoms, functioning), provider-level factors (e.g., level of professional experience, attitudes), intervention-specific characteristics (e.g., therapeutic modality), service delivery characteristics (e.g., frequency of sessions, location of care), organizational influences (e.g., culture and climate), and environmental factors (e.g., service system financing policies). The traditions of interventions and services research have led to differences in their relative emphases on the various aspects of this multilayer context. Interventions research has most traditionally focused on client, therapist, and intervention characteristics, whereas services research has focused primarily on service delivery parameters, organizational characteristics, and environmental factors. Moreover, although both traditions focus on client-level outcomes, intervention science tends to emphasize changes in symptom severity and diagnostic status, whereas services research examines changes in consumer-perceived functioning (e.g., quality of life). There is not a distinct polarization between the two types of research, but they also seem to fall upon two continua of foci: internal versus external validity and degree of diagnostic heterogeneity. Interventions science generally prioritizes internal validity with relatively homogenous target populations. Services research often emphasizes heterogeneity and real-world clinical population representation in an effort to maximize external validity.

The goal of this series of articles is to demonstrate how the strengths of the interventions and services research paradigms, both in terms of their focus and in their methodologic traditions, can inform one another. There are many ways in which the interaction between interventions and services research might shape future research studies or inform current lines of research. Scientific advances that such collaboration might offer include (a) mixed methodologic approaches (e.g., anthropologic and clinical trial approaches), (b) outcome measures that target multiple layers of the mental health service delivery context (e.g., use of symptom, quality of life, and organizational indicators), (c) scientific understanding of the interactions between care delivery context layers (e.g., how provider or organizational characteristics impact client outcomes), and (d) enriched compositions of the research teams that include not only diverse scientific disciplines but also relevant stakeholder groups (e.g., provider, consumers).
ENHANCING THE UTILITY AND GENERALIZABILITY OF CLINICAL RESEARCH

Various authors have articulated the distinction between efficacy and effectiveness research (cf. Barlow, 1996; Nathan, Stuart, \& Dolan, 2000). The lines between these two types of research are not entirely clear, and controversy about their specific definitions remains (Donenberg, Lyons, \& Howard, 1999; Wells, 1999). Generally speaking, however, efficacy research involves (a) a highly specified treatment intervention, (b) a well-specified patient population, and (c) highly controlled conditions. Often, efficacy research also involves a RCT design. On the other hand, effectiveness research involves (a) some version of a known-efficacious treatment, (b) a more heterogeneous patient population, (c) conditions that approximate usual care (cf. Wells, 1999), and (d) application of a variety of research designs including, but not limited to, the RCT design (benchmarking strategy; e.g., Wade, Treat, \& Stuart, 1998). Another distinction drawn by Rounsaville and colleagues (e.g., Carroll \& Rounsaville, 2003; Rounsaville, Carroll, \& Onken, 2001) is that efficacy research involves initial safety and feasibility testing, whereas effectiveness research must "address issues of transportability of treatments whose efficacy has been demonstrated" (Carroll \& Rounsaville, 2003, p. 334). In sum, efficacy research emphasizes internal validity, whereas effectiveness research emphasizes external validity.

Discussions of the efficacy-effectiveness distinction often emphasize the limitations of the traditional "academic" or "regulatory-model" RCT for informing clinical practice and policy (e.g., Hoagwood, Hibbs, Brent, \& Jensen, 1995; Hogarty, Schooler, \& Baker, 1997; Norquist, Lebowitz, \& Hyman, 1999; Southam-Gerow, 2004). These arguments contend that by loosening inclusion criteria, incorporating more diverse providers and settings, and employing outcome measures that tap domains such as disability, service use, and costs, results of intervention trials will be of greater utility to stakeholders, including consumers, providers, payers, and policy makers. By virtue of the fact that they incorporate the very factors that challenge or facilitate the work of typical providers, intervention studies conducted in more naturalistic settings will no doubt speed up the process of identifying transportable interventions and better achieve the dual aims of optimizing therapeutics as well as service delivery. 
This set of articles echoes the need for more stakeholder-relevant interventions research. However, with the articles in this special issue, we are hoping to direct attention beyond this focus, toward collaborations between interventions and services scientists that take many forms. First, our goal is broader in that the discussion of this set of articles is not limited to intervention studies that focus on a particular intervention that targets a particular population. Rather, we seek to encourage opportunities to embed services questions in interventions research (e.g., in RCTs, wherever they are conducted) more generally. In this manner, the results of these studies might contribute to the services research literature and the knowledge base more globally, in addition to informing questions about a particular intervention. Moreover, our discussion is not limited to opportunities for services research to inform interventions research; we also highlight the potential value of a more explicit interventions research viewpoint in conducting services research. Thus, our goal is not only to encourage more services-relevant interventions research, but also more interventions-relevant services research.

\section{ARTICLES IN THE SERIES}

The articles that follow this article describe recent crossfertilization research efforts by a multidisciplinary group of researchers whose work exemplifies the sort of crossfertilization efforts we advocate. We are here using the term cross-fertilization to mean research that integrates aspects of interventions and services science. These scientists represent the fields of nursing, psychiatry, social work, and clinical, cognitive, and industrial/organizational psychology. Following these articles, commentaries by interventions and services researchers reflect on the potential of such integrative efforts to improve the quality of new scientific efforts and ultimate sustainability of effective mental health therapeutics and care delivery. As an introduction to the articles in this series, we now highlight how each illustrates an innovative integration of interventions and services research.

\section{Using Interventions Science Techniques to Improve Our Understanding of Typical Clinical Practice}

Until relatively recently, much practice research has focused on quantifying services received and, to a lesser extent, on describing the quality of these services. In contrast, there have been relatively few efforts to unpack the "black box" of usual care or to test conceptually derived hypotheses regarding how variations in usual care practices impact patient outcomes. Garland, Hurlburt, and Hawley (this issue) describe how they have used interventions research approaches and strategies, including a detailed psychotherapy process coding system, to achieve a more finely grained characterization of psychotherapeutic interventions for children with disruptive behavior disorders in community outpatient clinic settings. In addition, their work examines the extent to which usual care is consistent with evidence-based practice principles, and it addresses hypotheses regarding the degree of overlap with evidence-based practices and patient outcomes. Their article details the investigators' use of interdisciplinary approaches (e.g., qualitative approaches to characterize provider-defined principles of care) and describes the process of establishing collaborative relationships with the providers themselves as research partners in setting the research agenda and conducting the research. Finally, the authors discuss future directions and comment on the potential of this work for informing our understanding of usual care practices; elucidating the patient-, provider-, dyadic-, and organization-level factors that are related to outcomes in naturalistic settings; identifying novel strategies that might inform treatment development; and ultimately, facilitating the implementation of research- and provider-defined principles of care that are associated with good outcomes.

\section{Employing the RCT Template to Address Questions About Real-World Service Implementation}

As noted earlier, RCTs have been traditionally used to address patient-level intervention outcomes in highly controlled settings, whereas broader questions regarding the delivery of services have been pursued separately, often in a more descriptive fashion. The article from Zatzick, Simon, and Wagner (this issue) details efforts to use the RCT as a platform, not only for addressing intervention outcomes, but also for embedding servicerelevant questions in emergent- and primary-care settings. The fact that these investigators work in nonmental health settings has necessitated flexibility. The authors have used creative modifications to the typical RCT in terms of the participant identification strategies, interventionists, service delivery models, study designs, and 
definitions of outcome that are employed. In part, because their work addresses interventions in general medical settings, their research programs naturally evolved in a multidisciplinary context that has included teams of various physicians, nurses, and other medical professionals. But beyond that, their article illustrates their efforts to maximize input from various social science disciplines, including psychology, clinical epidemiology, and economics. Finally, the authors reflect on how variations on RCT methods and various interdisciplinary collaborations might be further used to understand potential leverage points for optimizing interventions and their delivery.

\section{Understanding the Impact of Patient and Provider Preferences}

Scientific attention to consumer and provider preferences is growing; however, little is known about the impact of these preferences on service delivery engagement or intervention outcomes. In addition, research on how clients or providers make treatment choices is often done in isolation from knowledge gained through the basic decision-making sciences. Furthermore, basic decision-making measurement strategies, often analog in nature, are difficult to translate into dynamic care delivery settings. The ongoing work conducted by Drs. Wills and Holmes-Rovner marries concepts drawn from basic decision theories with the real-world dynamic nature of mental health patient and provider decision making. Using their interdisciplinary expertise in nursing and cognitive psychology, the authors define a theoretical framework to better understand the way in which a mental health service delivery context might influence a client's care preferences, decisions, behaviors, and eventually outcomes. Starting with their own emerging work on decision aids, the authors draw important implications of client and provider preferences for intervention delivery, client and provider behavior change, as well as future research measurement strategies and design.

\section{Integrating the Constructs of Organizational Culture and Climate into Services and Interventions Research}

Emerging evidence suggests that the service delivery context is influential in determining the successful implementation of efficacious interventions and in achieving positive outcomes. Despite this, relatively little scientific research has examined the interaction between clinical interventions and the context in which clinical care is delivered. Using their expertise in organizational development and mental health service systems, Hemmelgarn, Glisson, and James (this issue) focus their work on the role of organizational culture and climate, particularly within child welfare systems. Within the field of children's mental health, their scientific work was some of the first to substantiate empirical relationships between organizational constructs and care quality, as well as child outcomes. In this article, they define these constructs, describe the way in which they have tested their influence on mental healthcare delivery, and describe their development of a new organizational intervention specifically designed for the social service care delivery sector. The authors offer suggestions for ways in which either interventions or services researchers might include examinations of organizational culture or climate or both within future research studies.

\section{Integrating Interventions and Services Research: Other Examples}

The articles in this series comprise a select but not exhaustive set of research efforts that address the interface of services and interventions research. In each article, the authors discuss potential implications of their research and promising future directions, including additional opportunities for cross-fertilization between interventions and services research. Some additional examples of ongoing research that spans the boundaries of services and interventions research are briefly illustrated in succeeding section. Please note that most of the research we discuss next is in the early stages and thus serves only as illustrations of the hypotheses and methods. Our discussion is meant to provide exemplars of ways that the interventions and services research paradigms might be combined.

\section{Improving Outcome Measurement}

Services research methods and sensibilities hold promise for advancing state-of-the-art outcome measurement in interventions research. Outcome assessment in traditional efficacy research can be criticized as being at once too narrow (focused on symptomatic or diagnostic status while ignoring other important functional domains; e.g., Norquist et al., 1999) and yet too extensive (char- 
acterized by the inclusion of multiple, often overlapping, complicated instruments). Therefore, these existing measurement strategies are usually not ideally suited for interventions research in naturalistic settings. Services research approaches might be used to identify outcome domains of relevance to consumers, providers, and policy makers. Alternatively, these approaches might be used to develop practice- and consumer-friendly instruments to assess more typically studied symptom-level outcomes. For example, a currently funded career development award focuses on the use of cognitive science, cognitive interviewing, and qualitative research techniques to inform the development and refinement of valid and efficient outcome measures for use in the full range of practice settings and patient populations (MH064073, M. Shumway, principal investigator [P.I.]; e.g., Shumway, Chouljian, \& Battle, 2005).

\section{Designing Culturally Relevant Interventions}

The general applicability of interventions research to diverse populations has been questioned (e.g., National Advisory Mental Health Council, 2001; Chorpita et al., 2002). Services research might be used to inform the development and refinement of interventions that better meet the service needs of ethnic and other minority consumers. Currently, several efforts are underway to use observational, survey, and qualitative research techniques to identify culturally salient features of disorders and to inform culturally and socially valid interventions in order to enhance client engagement and retention, facilitate intervention implementation, and improve outcomes for ethnic minority consumers. Examples include efforts to adapt evidencebased interventions for use with Asian American families affected by physical abuse and Mexican American families presenting for treatment of disruptive behavior disorders (MH066864, A. Lau, P.I. and MH001924, K. McCabe, P.I., respectively; e.g., Yeh, Hough, McCabe, Lau, \& Garland, 2004).

\section{Facilitating Dissemination and Implementation}

Other ongoing studies capitalize on services research and clinical trial methods in an effort to further our understanding of how to successfully convey information to providers (i.e., dissemination) and how to promote provider behavior change (i.e., implementation and sustained use of new practices). For example, an ongoing study examines the implementation of an evidence-based practice designed to enhance parenting skills for parents in the foster care system (MH060196, P. Chamberlain, P.I.). The study employs a cascading dissemination model where, in the first wave, the intervention developers train and supervise staff in the field to implement the intervention. In the second wave, the involvement of the original developers is reduced and the newly trained staff members are responsible for training and supervising the next set of interventionists (e.g., Chamberlain \& Smith, 2005). Client, provider, and organizational factors that impact the fidelity with which the intervention is implemented in both waves of intervention are assessed. Another study is examining intra- and extraorganizational factors that relate to the transportability of Multisystemic Therapy (MST), a comprehensive, multicomponent family-based intervention that has been demonstrated to improve child outcomes and family functioning in RCTs with youth with serious problems (e.g., violent and chronic juvenile offenders, sex offenders; MH059138, S. Schoenwald, P.I.). This study examines child outcomes with MST delivered by multiple clinicians in usual care settings across multiple localities, and tests whether the impact of clinician variables and organizational factors on intervention outcomes is mediated by clinician adherence to the MST model.

\section{Improving Quality of Care}

Methods more commonly used in traditional services research (e.g., qualitative methods, analysis of service utilization data/patterns) can also be used to identify mutable parameters of service delivery that can be targeted in interventions aimed at improving quality of care. An ongoing study uses more traditional interventions and services research strategies in a sequential, iterative fashion to design and test interventions that will ultimately enhance the quality of care and outcomes for depressed adolescents (MH001992, T. Kramer, P.I.). The first phase of this research uses structured interviews with providers to determine factors that interfere with appropriate identification and treatment of depressed adolescents in mental health specialty care settings. This research then forms the basis for the development of a quality improvement intervention that is later tested within a pilot RCT to examine its feasibility and effectiveness for enhancing care quality and youth outcomes. Qualitative 
methods, including follow-up interviews with providers and consumers, are then used to identify barriers to quality enhancement efforts and to inform further revisions to the quality improvement intervention.

\section{FUTURE DIRECTIONS}

As a new generation of mental health researchers emerges, we are encouraged to see the increasing synergy between services and interventions research conceptualizations and methodologies. A shared interventions and services research agenda holds promise for overcoming some unnecessary field barriers. Such barriers include documented high treatment dropout rates and poor client engagement in mental health interventions, efficacy ceilings and limited durability of treatment gains with even our most promising interventions, limitations in our ability to promote provider behavior change or sustain it or both, and slow progress at identifying truly novel therapeutic techniques. Through this special issue, we hope to illustrate the possibilities and promise of interventions and services research collaboration, and importantly, to challenge future work in this area to aim high. Such pursuits carry with them a high level of scientific challenge but considerable potential for great public health impact.

\section{ACKNOWLEDGMENT}

The views expressed within this article represent those of the authors and are not intended to represent the NIMH, NIH, or DHHS or Virginia Commonwealth University. Preparation of this article was supported in part by National Institute of Mental Health grant K23 MH69421.

\section{REFERENCES}

Barlow, D. H. (1996). Health care policy, psychotherapy research, and the future of psychotherapy. American Psychologist, 51, 1050-1058.

Burns, B. J., Costello, E. J., Angold, A., Tweed, D., Stangl, D., Farmer, E. M., et al. (1995). Children's mental health service use across service sectors. Health Affairs, 14(3), 147-159.

Carroll, K. M., \& Rounsaville, B. J. (2003). Bridging the gap: A hybrid model to link efficacy and effectiveness research in substance abuse treatment. Psychiatric Services, 54, 333-339.

Chamberlain, P., \& Smith, D. K. (2005). Multidimensional treatment foster care: A community solution for boys and girls referred from juvenile justice. In E. D. Hibbs \& P. S. Jensen (Eds.), Psychosocial treatments for child and adolescent disorders: Empirically based strategies for clinical practice (2nd ed., pp. 557-573). Washington, DC: American Psychological Association.

Chambless, D. L., \& Ollendick, T. H. (2001). Empirically supported psychological interventions: Controversies and evidence. Annual Review of Psychology, 52, 685-716.

Chorpita, B. F., Yim, L. M., Donkervoet, J. C., Arensdorf, A., Amundsen, M. J., McGee, C., et al. (2002). Toward largescale implementation of empirically supported treatments for children: A review and observations by the Hawaii Empirical Basis to Services Task Force. Clinical Psychology: Science and Practice, 9, 165-190.

Donenberg, G. R., Lyons, J. S., \& Howard, K. I. (1999). Clinical trials versus mental health services research: Contributions and connections. Journal of Clinical Psychology, 55, 11351145.

Estroff, S. (1981). Making it crazy: An ethnography of psychiatric clients in an American community. Berkeley and Los Angeles: University of California Press.

Friedman, L. M., Furberg, C. D., \& DeMets, D. L. (1998). Fundamentals of clinical trials (3rd ed.). New York: Springer-Verlag.

Hoagwood, K., Burns, B. J., Kiser, L., Ringeisen, H., \& Schoenwald, S. J. (2001). Evidence-based practice in child and adolescent mental health services. Psychiatric Services, 52, 1179-1189.

Hoagwood, K., Hibbs, E., Brent, D., \& Jensen, P. (1995). Introduction to the special section: Efficacy and effectiveness in studies of child and adolescent psychotherapy. Journal of Consulting and Clinical Psychology, 63, 683-687.

Hogarty, G. E., Schooler, N. R., \& Baker, R. W. (1997). Efficacy versus effectiveness. Psychiatric Services, 48, 1107.

Hollingshead, A. B., \& Redlich, F. C. (1958). Social class and mental illness: A community study. New York: Wiley.

Kataoka, S. H., Zhang, L., \& Wells, K. B. (2002). Unmet need for mental health care among U.S. children: Variation by ethnicity and insurance status. American Journal of Psychiatry, 259, 1548-1555.

Kazdin, A. E., \& Weisz, J. R. (Eds.). (2003). Evidence-based psychotherapies for children and adolescents. New York: Guilford Press.

Kendall, P. C. (1998). Empirically supported psychological therapies. Journal of Consulting and Clinical Psychology, 66, 3-6.

Kessler, R. C., Berglund, P. A., Bruce, M. L., Koch, J. R., Laska, E. M., Leaf, P. J., et al. (2001). The prevalence and correlates of untreated serious mental illness. Health Services Research, 36, 987-1007.

Leaf, P. J., Alegria, M., Cohen, P., Goodman, S. H., Horwitz, S. M., Hoven, C. W., et al. (1996). Mental health service 
use in the community and schools: Results from the fourcommunity MECA Study. Methods for the Epidemiology of Child and Adolescent Mental Disorders Study. Journal of the American Academy of Child and Adolescent Psychiatry, 35, 889-897.

Mechanic, D. (1989). The evolution of mental health services and mental health services research. In C. A. Taub, D. Mechanic, \& A. Hohmann (Eds.), The future of mental health services research. DHHS Publication Number (ADM) 89-1600. Washington, DC: Suppt Of Docs, U.S. Government Printing Office.

Nathan, P. E., \& Gorman, J. M. (2002). A guide to treatments that work (2nd ed.). London: Oxford University Press.

Nathan, P. E., Stuart, S. P., \& Dolan, S. L. (2000). Research on psychotherapy efficacy and effectiveness: Between Scylla and Charybdis? Psychological Bulletin, 126, 964-981.

National Advisory Mental Health Council Workgroup on Child and Adolescent Mental Health Intervention Development and Deployment. (2001). Blueprint for change: Research on child and adolescent mental health. Washington, DC: U.S. Department of Health and Human Services.

National Institutes of Health, \& National Institute of Mental Health. (1999). Bridging science and service: A report by the National Advisory Mental Health Council's Clinical Treatment and Services Research Workgroup. Retrieved from http://www.nimh.nih.gov/publicat/nimhbridge.pdf.

Norquist, G., Lebowitz, B., \& Hyman, S. (1999). Expanding the frontier of treatment research. Prevention \& Treatment, 2, np.

Ringel, J. S., \& Sturm, R. (2001). National estimates of mental health utilization and expenditures for children in 1998. Journal of Behavioral Health Services \& Research, 28, 319-333.

Rounsaville, B. J., Carroll, K. M., \& Onken, L. S. (2001). A stage model of behavioral therapies research: Getting started and moving on from stage I. Clinical Psychology: Science and Practice, 8, 133-142.

Schoenwald, S. K., \& Hoagwood, K. (2001). Effectiveness and dissemination research: Their mutual roles in improving mental health services for children and adolescents. Report on Emotional and Behavioral Disorders in Youth, 2, 3-4, 18-20.

Shumway, M., Chouljian, T. L., \& Battle, C. L. (2005). Measuring preferences for schizophrenia outcomes with the time tradeoff method. Journal of Behavioral Health Services $\mathcal{E}$ Research, 32, 14-26.

Southam-Gerow, M. A. (2004). Some reasons that mental health treatments are not technologies: Toward treatment development and adaptation outside labs. Clinical Psychology: Science and Practice, 11, 186-189.

Street, L. L., Niederehe, G., \& Lebowitz, B. (2000). Toward greater public health relevance for psychotherapeutic interventions research: An NIMH workshop report. Clinical Psychology: Science and Practice, 7, 127-137.

Wade, W. A., Treat, T. A., \& Stuart, G. L. (1998). Transporting an empirically supported treatment for panic disorder to a service clinic setting: A benchmarking strategy. Journal of Consulting and Clinical Psychology, 66, 231-239.

Wang, P. S., Berglund, P. A., \& Kessler, R. C. (2003). Patterns and correlates of contacting clergy for mental disorders in the United States. Health Services Research, 38, 647-673.

Weiss, B., Catron, T., \& Harris, V. (2000). A 2-year follow-up of the effectiveness of traditional child psychotherapy. Journal of Consulting and Clinical Psychology, 68, 1094-1101.

Weisz, J. R., Weiss, B., \& Donenberg, G. R. (1992). The lab versus the clinic: Effects of child and adolescent psychotherapy. American Psychologist, 47, 1578-1585.

Wells, K. B. (1999). Treatment research at the crossroads: The scientific interface of clinical trials and effectiveness research. American Journal of Psychiatry, 156, 5-10.

Westen, D., Novotny, C. M., \& Thompson-Brenner, H. (2004). The empirical status of empirically supported psychotherapies: Assumptions, findings, and reporting in controlled clinical trials. Psychological Bulletin, 130, 631-663.

The WHO World Mental Health Survey Consortium. (2004). Prevalence, severity, and unmet need for treatment of mental disorders in the World Health Organization World Mental Health Surveys. Journal of the American Medical Association, 291, 2581-2590.

Yeh, M., Hough, R. L., McCabe, K., Lau, A., \& Garland, A. (2004). Parental beliefs about the causes of child problems: Exploring racial/ethnic patterns. Journal of the American Academy of Child \& Adolescent Psychiatry, 43, 605-612.

Received January 27, 2005; revised June 27, 2005; accepted June 28, 2005. 